\title{
Survey on Recent Reliable Routing protocols in WSN
}

\author{
Abhay K. Meshram1, Prof. Deepak C. Mehetre ${ }^{2}$ \\ Department of Computer Engineering, K.J. College of Engineering and Management Research, Pune ${ }^{1,2}$
}

\begin{abstract}
In wireless sensor network (WSN) technology has offered the accessibility of small as well as low-cost sensor nodes with capability of sensing different types of physical and environmental conditions, data processing, and wireless communication. Various types of sensing capabilities results in large amount of application domain. Moreover, the characteristics of wireless sensor networks need more effective techniques for data forwarding along with processing. In WSN, the sensor nodes have a limited transmission range, and their processing and storage capabilities as well as their energy resources are also limited. For managing the routes in the network and to ensure reliable multihop interaction under these conditions routing protocols in wireless sensor networks are responsible. This paper, studies the different types of recent routing protocols in Wireless Sensor Network and compare their strengths and limitations.
\end{abstract}

Keywords: Wireless sensor networks, routing protocols, energy efficient, multi hop, data forwarding.

\section{INTRODUCTION}

The rapid development in the technology has lead to different ways which lead to the creation of various type of networks for several purposes. The components such as collection of data, interpret and act on real time data gains increasing interest. Moreover, for the collection of data in wired sensor networks have many of factors which do not simple the process of doing so.

The advantage of this is fast development along with increasing use of Wireless sensor networks (WSN).This are one kind of connection network that offers redundant and limited fault tolerant wireless connection between sensors, actuators and collectors. Generally, WSNs are a massive combination of sensor nodes which are used to protect and flexible networks by combining the sensor radio and the CPU. The WSNs works more efficiently and also are low power utilization networks. In any type of networks, energy is the fundamental resource as the networks are operated to exhibit certain kind of properties there is a definite need of optimization of the network architecture in order to reducing the resource consumed. The WSNs are a mixture of distinct routing, power management and data distribution protocols. The protocols used here require to support different constraints which are useful in maintaining and proper functioning of the WSNs.

The basic goals of a WSN are to:

(i) Examine the value of physical variables at a given location.

(ii) Identify the occurrence of events of interest, and calculate parameters of the detected event or events.

(iii) Classify a recognize object, and

(iv) Track an object.

The important requirements of a WSN are:

(i) use of a large number of sensors,

(ii) attachment of stationary sensors,

(iii) low energy consumption,

(iv) self organization capability,

(v) collaborative signal processing,

(vi) querying ability

The different type of constraints or factors to be considered are memory, power consumption, scalability, adaptively, latency, tolerance also the size of the sensor include in it. The implementation of these protocols helps in proper working of the WSNs as these were perfectly done sensor networks are having number of applications in different fields. The different type of applications are military sensing, physical security, air traffic control, traffic surveillance, video surveillance, industrial and manufacturing automation, distributed robotics, environment monitoring, and building and structures monitoring, Medical applications, atmospheric observance, home intelligence etc. 
To find out a path between the source node and destination node the method named as Routing. For this purposes a device is used, called router. With the help of router the message or packet can be sent to move from one node to another node and reach to the destination. In routing mechanism, a routing table is used to finding the best path \& sends the message or packet over the path. With the help of these routing table's intermediate nodes passes the data. But routing is preferred at high level because it is executed with software and a very complex process. Because analyzing the best path for the packets to be transmitted is preferred with the help of routing table.

Generally, routing in WSNs can be divided into 3 types of routing flat-based routing, hierarchical-based routing, and location-based routing depend on the network structure. In flat-based routing, all nodes are typically assigned equal roles or functionality. In hierarchical-based routing, however, nodes will play different roles in the network. In location-based routing, sensor nodes' positions are exploited to route data in the network. A routing protocol is considered adaptive if certain system parameters can be controlled in order to adapt to the current network conditions and available energy levels. Moreover, these protocols can be categorized into multipath-based, query-based, negotiation-based, QoS-based, or coherent-based routing techniques depending on the protocol operation. In addition to the above, routing protocols can be divided into three categories, namely, proactive, reactive, and hybrid protocols depend on how the source finds a route to the destination. In proactive protocols, all routes are estimated before they are really needed, while in reactive protocols, routes are computed on demand. Hybrid protocols use a combination of these two ideas. Many other protocols rely on timing and position information. It also shed some light on these types of protocols in this paper. In order to streamline this survey, use a classification according to the network structure and protocol operation (routing criteria).

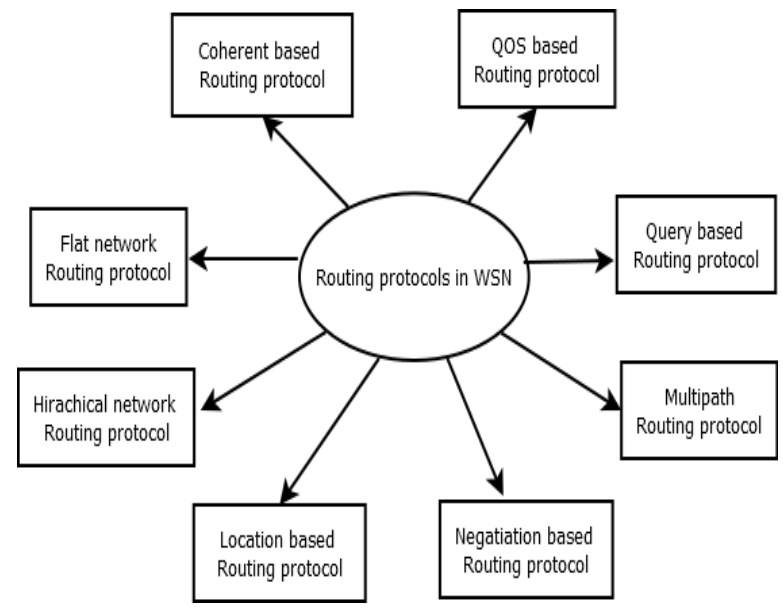

Fig 1: Routing Protocol in WSN

\section{Challenges:}

Some of the factors that affect the routing protocols are:

1. Node deployment: Node deployment in WSNs is application dependent and affects the performance of the routing protocol. The deployment can be either deterministic or randomized..

2. Energy consumption: One of the main component that affect the sensor networks is the low power availability. The energy available for the sensor is depend upon the battery life time. For the transmission of the signal multi hop routing will be of more significance as it consumes less power than direct communication.

3. Network designing: As the sensors are connected through a wireless connection it is essential to recognize the proper routing and the network topology to be used. Also the message traffic is also to be considered at each node as it may lead to the instability of the system if more traffic is present at similar node.

4. Data transfer: The main objective in the WSNs is the proper transfer of data from the sensors to the connected device. This can be done only when a proper routing is applied amongst the network.

5. Tolerance and scalability: If any sensor or node gets failure due to loss in power or any other external factors it should not affect the entire network as this may lead to total malfunction. Also irrespective of the no. of sensor in the network the routing scheme must be scalable to respond to the events.

\section{LITERATURE REVIEW}

This paper [1], enhance the energy hole problem of Receiver-Centric MAC(RC-MAC), entitled as EERC-MAC, which has greater energy conservation as well as better throughput than RC-MAC. This proposed EERC-MAC offers fairness 
between source nodes without sacrificing the throughput. It uses a beacon method that lets nodes to get sleep appropriately between data transmission and change the data routing path to ignore energy hole. The experimental results prove that the throughput is extensively developed by receiver-centric scheduling. For better energy efficiency in nodes battery the improved throughput used.

This paper [2], offered a new handshake scheme in the Data Forwarding Phase (DFP) of the RAEED protocol. The new protocol RAEED-NEHA is proved to be more resilient towards DoS attacks like black hole attacks, sink hole attacks etc. The proof is established using the methodology introduced in earlier research which is a mixture of formal verification, computer aided simulation and observance of response of the protocol running on MicaZ motes. The use of formal methods has ensured the absence of hidden bugs or boundary condition scenarios present in the RAEEDNEHA protocol.

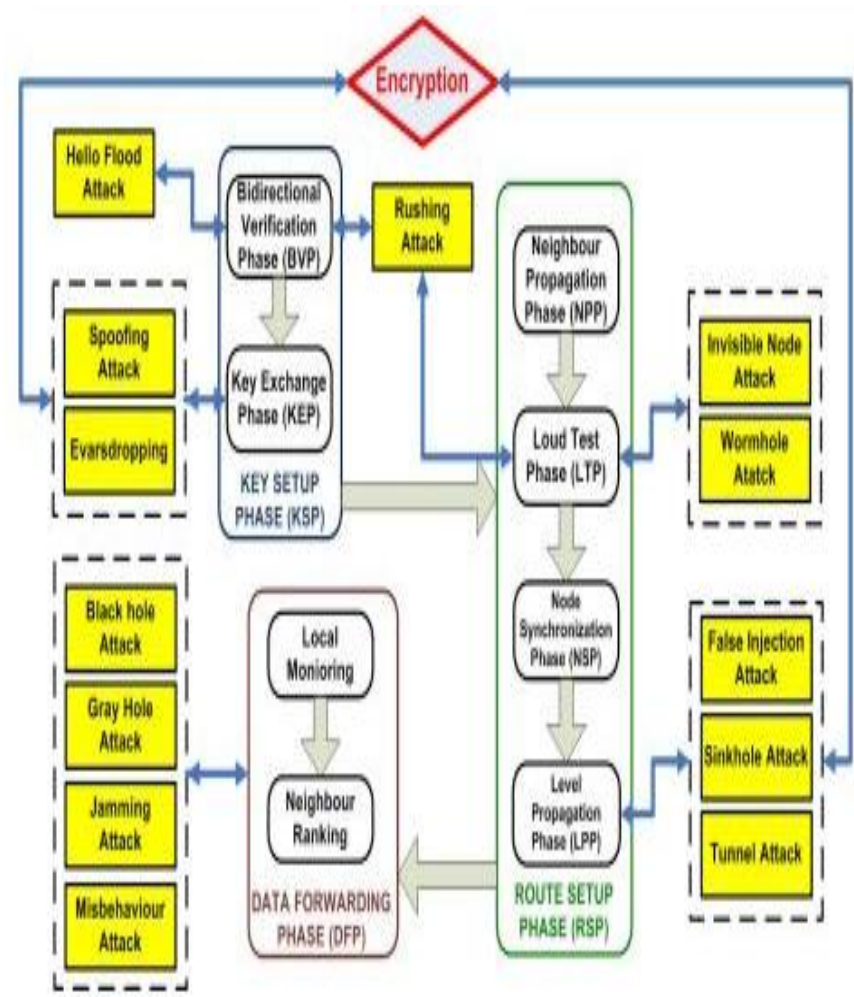

Fig.2. RAEED-NEHA Protocol Phases [2]

This paper [3], implemented an artificial neural network based energy-efficient as well as robust routing schema for WSNs named as ELDC. In this approach, the network is trained on large data set including almost all scenarios to make the network more reliable and adaptive to the environment. Moreover, it helps group based methodology to enlarge the lifetime of the entire network, where groups may have distinct sizes. An artificial neural network provides an efficient threshold values for the selection of a group's chief node and a cluster head based on back propagation technique and allows intelligent, efficient, and robust group organization. This proposed method is extremely energy-efficient capable to increase sensor nodes' lifetime. The experimental results display that it outperforms LEACH protocol by $42 \%$, and other state-of-theart protocols by more than $30 \%$.

This paper [4], proposed a new approach to enlarge the network lifetime depend on the Improved Particle Swarm Optimization algorithm, which is an optimization method planned to select target nodes. The protocol takes into account both energy efficiency as well as transmission distance, and relay nodes are used to improve the excessive power consumption of the cluster heads. The proposed protocol results in better distributed sensors and a well-balanced clustering system improving the network's lifetime. It compare the proposed protocol with comparative protocols by varying a number of parameters, e.g., the number of nodes, the network area size, and the position of the base station. The experimental result show that the proposed protocol executes well against other comparative protocols in different scenarios.

This paper [5], proposed on LEACH based energy efficient routing protocol for wireless Sensor networks. Depend on both particle swarm optimization technique as well as V-LEACH protocol a method of routing is suggested. 


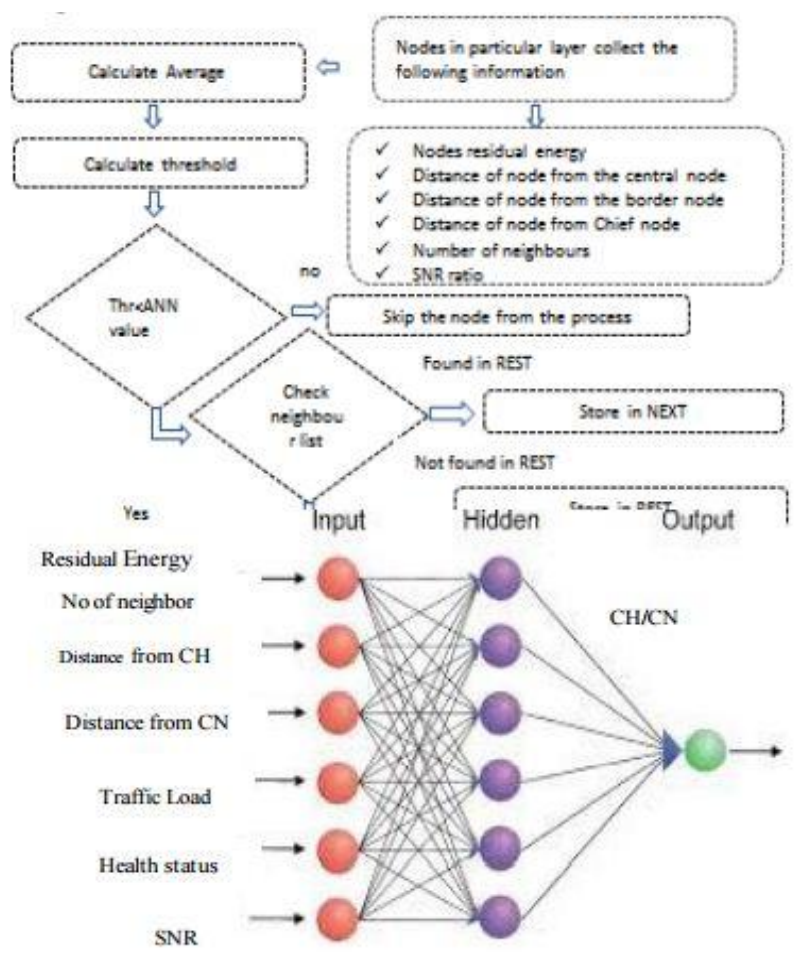

Fig.3. Chief node and Cluster head selection process [3]

It analyzed performance metrics Data Transmitted, Consumed energy, End to End delay for comparison, performance comparison shows proposed protocol offer better performance. Energy consumed in proposed protocol is less in comparison to existing protocol, delay time in proposed protocol is less in comparison to existing protocol, also no of packets required to send same amount of data is less in proposed protocol with compared to previous protocol.

This paper [6], proposed a routing protocol named as Grid Clustering Hierarchy $(\mathrm{GCH})$ that offers an efficient energy management for WSNs. This protocol split the network into a variable number of virtual grids depend on the current average energy of the network to create optimum clusters in terms of energy utilization. By using a standard radio energy dissipation model that is generally used for simulation of WSNs, GCH is simulated and its execution is compared with a well-known routing protocol for WSNs named LEACH.

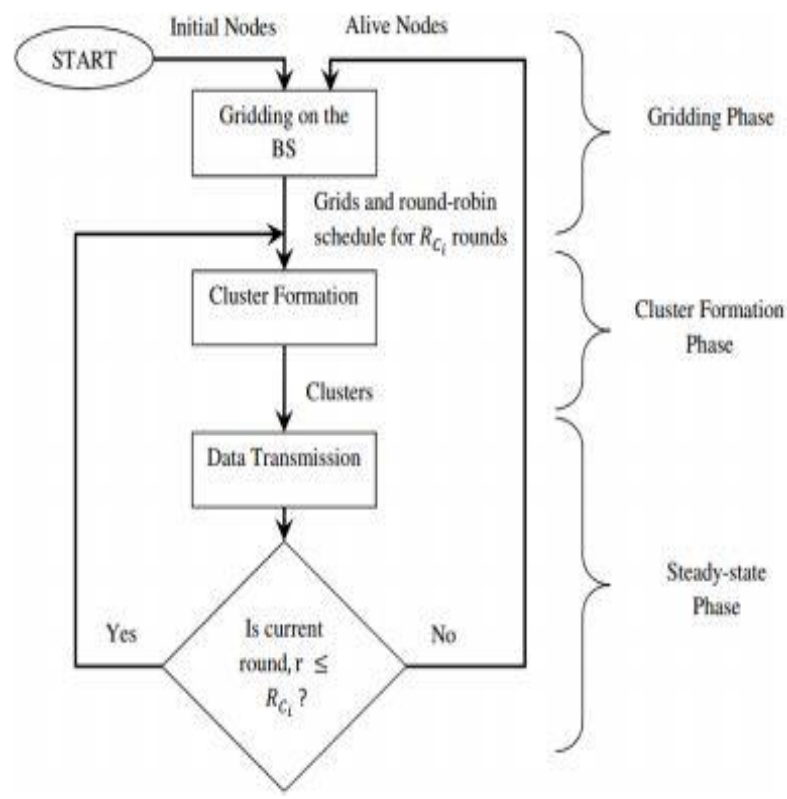

Fig. 4: Flowchart of the operations of GCH [6] 
In [7] a Location based Topology Control (LTC) is used to manage the operation of the active nodes through the sensing field. Hence the Intra term is reduced. Additionally, four multiple levels of clustering hierarchy (FL-LEACH) are occupied. Actually, FL-LEACH is an extended work for three layers clustering (TL-LEACH). Both of them are depend on restricting the number of nodes which can interact directly with BS. This minimizes and balances the Interterm. The experimental results show that this proposed protocol is highly efficient than other conventional protocols, such as LEACH and TL-LEACH.

In this paper [8], it optimized the routing path in IECBR (Improved Energy Efficient Chain Based Routing) protocol which is one of the most recent techniques depend on PEGASIS (power Efficient Gathering in Sensor Information System). It used Honey Bee Optimization (HBO) method to select the next node which is to be added for the chain construction for the data transmission from the network to the base station. The energy and distance are the two parameters which are exploited as optimization parameters. From the experimental results that the H-IECBR (Honey bee optimization based IECBR) improves the first node dead by $56 \%$ and network lifetime by $116 \%$ as compare to the IECBR. The proposed protocol H-IECBR is favorite to the applications where the network area is not scalable due to some inevitable constraints.

In this paper [9], the network lifetime of Robust Formally Analyzed Protocol for Wireless Sensor Networks Deployment (RAEED) is developed by introducing a new version of RAEED called RAEED with energy-awarerouting (RAEED-EA). Energy aware routing needs suitable changes in the third and final phase i.e. Data Forwarding Phase of RAEED. In RAEED the decision of selecting the next node was overall depend on the throughput of the target nodes, whereas in RAEED-EA the decision is taken based upon residual energy and throughput of the target nodes. Formal verification is used for performance evaluation and these protocols are compared in terms of minimum network life-span. Results show that by changing the network topology the improvement in network lifetime of a network using RAEED-EA over RAEED ranges from 3 percent to 40 percent.

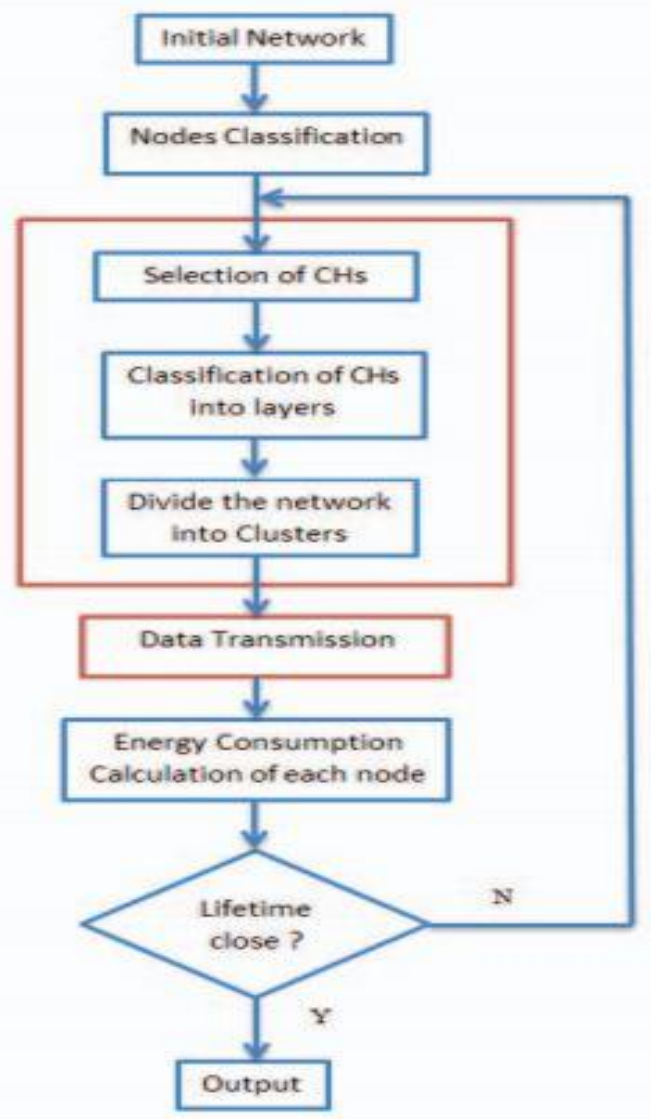

Fig.5:.LTC flowchart [7]

In this paper[10], assume Base station is installed out of the sensing field and numbers of gateway nodes are deployed at the edge of sensing field. These gateway nodes are rechargeable therefore, it minimize cost. It also reduces traffic issues and minimizes distance for reliable transmission of data. The experimental results shows that the proposed 
gateway based protocol is better in terms of network lifetime than traditional protocol like SEP protocol by showing significant improvement in terms of number of half dead nodes, dead nodes, alive nodes and also in terms of remaining energy of sensor node. It also observe that in Gateway based SEP or G-SEP Protocol Throughput is increased in case of packets transmit to $\mathrm{CHs}$ and packets transmit to BS respectively over traditional SEP Protocol.

Table 1: Survey Table

\begin{tabular}{|c|c|c|c|c|}
\hline S.No & Title & Implemented Protocols & Advantages & Disadvantages \\
\hline 1. & $\begin{array}{l}\text { EERC-MAC: Energy efficient } \\
\text { Receiver Centric MAC protocol for } \\
\text { Wireless Sensor network }\end{array}$ & $\begin{array}{l}\text { Proposed EERC-MAC } \\
\text { protocol }\end{array}$ & Better Throughput & $\begin{array}{l}\text { Less energy } \\
\text { efficiency. }\end{array}$ \\
\hline 2. & $\begin{array}{l}\text { RAEED-NEHA: A new handshake } \\
\text { scheme for RAEED protoco }\end{array}$ & $\begin{array}{c}\text { Presented Data } \\
\text { Forwarding Phase (DFP) } \\
\text { of the RAEED protocol }\end{array}$ & More Resilient & $\begin{array}{l}\text { Affected by } \\
\text { various DOS } \\
\text { attack. }\end{array}$ \\
\hline 3. & $\begin{array}{l}\text { ELDC: An Artificial Neural } \\
\text { Network based Energy-Efficient } \\
\text { and Robust Routing Scheme for } \\
\text { Pollution Monitoring in WSNs }\end{array}$ & $\begin{array}{l}\text { Proposed ELDC } \\
\text { protocol. }\end{array}$ & $\begin{array}{l}\text { Extremely energy- } \\
\text { efficient and } \\
\text { capable to increase } \\
\text { sensor nodes } \\
\text { lifetime. }\end{array}$ & Securely routing \\
\hline 4. & $\begin{array}{l}\text { Clustering Hierarchy Protocol in } \\
\text { Wireless Sensor Networks Using an } \\
\text { Improved PSO Algorithm }\end{array}$ & $\begin{array}{l}\text { Implemented Clustering } \\
\text { Hierarchy Protocol }\end{array}$ & $\begin{array}{l}\text { Capable to enlarge } \\
\text { network lifetime. }\end{array}$ & $\begin{array}{l}\text { Energy is not } \\
\text { considered. }\end{array}$ \\
\hline 5. & $\begin{array}{l}\text { Energy efficient routing of WSN } \\
\text { using particle swarm optimization } \\
\text { and V-LEACH protocol }\end{array}$ & $\begin{array}{l}\text { Proposed on LEACH } \\
\text { based energy efficient } \\
\text { routing protocol }\end{array}$ & $\begin{array}{l}\text { Less energy } \\
\text { consumption. }\end{array}$ & $\begin{array}{l}\text { More time } \\
\text { consuming. }\end{array}$ \\
\hline
\end{tabular}

\section{CONCLUSION}

In this paper, the researcher analyzes the routing protocols in wireless sensor networks and classify them into many categories. Also given the advantages and drawbacks present in the various studies executed by various researchers. To deal with drawbacks in present systems we presented an idea of the new system.

\section{REFERENCES}

1. M. I. Khalil, M. A. Hossain, M. J. Haque and M. N. Hasan, "EERC-MAC: Energy efficient Receiver Centric MAC protocol for Wireless Sensor network," 2017 IEEE International Conference on Imaging, Vision \& Pattern Recognition (icIVPR), Dhaka, Bangladesh, 2017, pp. 1-5

2. Saghar, Kashif, Daniyal Yasin, and Ahmed Bouridane. "RAEED-NEHA: A new handshake scheme for RAEED protocol." Applied Sciences and Technology (IBCAST), 2017 14th International Bhurban Conference on. IEEE, 2017.

3. Mehmood, Amjad, et al. "ELDC: An Artificial Neural Network based Energy-Efficient and Robust Routing Scheme for Pollution Monitoring in WSNs." IEEE Transactions on Emerging Topics in Computing (2017).

4. Zhou, Yuan, Ning Wang, and Wei Xiang. "Clustering Hierarchy Protocol in Wireless Sensor Networks Using an Improved PSO Algorithm." IEEE Access 5 (2017): 2241-2253.

5. Singh, Alka, Shubhangi Rathkanthiwar, and Sandeep Kakde. "Energy efficient routing of WSN using particle swarm optimization and VLEACH protocol." Communication and Signal Processing (ICCSP), 2016 International Conference on. IEEE, 2016.

6. Amsalu, Seifemichael B., et al. "Energy efficient Grid Clustering Hierarchy (GCH) routing protocol for wireless sensor networks." Ubiquitous Computing, Electronics \& Mobile Communication Conference (UEMCON), IEEE Annual. IEEE, 2016

7. Fawzy, Abd Elwahab, et al. "Four-layer routing protocol with Location based Topology Control of active nodes in WSN." Computer Engineering \& Systems (ICCES), 2016 11th International Conference on. IEEE, 2016.

8. Gupta, Aman, et al. "H-IECBR: HBO based-Improved Energy Efficient Chain Based Routing protocol in WSN." Power Electronics, Intelligent Control and Energy Systems (ICPEICES), IEEE International Conference on. IEEE, 2016.

9. Khan, Naveed Ahmed, et al. "RAEED-EA: A formally analysed energy efficient WSN routing protocol." Applied Sciences and Technology (IBCAST), 2016 13th International Bhurban Conference on. IEEE, 2016.

10. Kaur, Harminder, Pallavi Jain, and Gaurav Prabhakar. "An advanced form of stable election multihop routing protocols using gateways in Wireless Sensor Networks." Computation of Power, Energy Information and Commuincation (ICCPEIC), 2016 International Conference on. IEEE, 2016. 